\title{
Hydromagnetic Flow over an Inclined Non-Linear Stretching Sheet with Variable Viscosity in the Presence of Thermal Radiation and Chemical Reaction
}

\author{
G. C. Shit ${ }^{1 \dagger}$ and S. Majee \\ ${ }^{I}$ Department of Mathematics Jadavpur University, Kolkata - 700032, India \\ ${ }^{2}$ Department of Applied Mathematics Indian School of Mines, Dhanbad, India \\ †Corresponding Author Email: gcs@math.jdvu.ac.in
}

(Received October 3, 2012; accepted July 9, 2013)

\begin{abstract}
An analysis has been made to investigate the effects of thermal radiation on the magnetohydrodynamic (MHD) flow and heat transfer over an inclined non-linear stretching sheet. The surface velocity of the stretching sheet and the transverse magnetic field are assumed to vary as a power function of the distance from the origin. The effect of internal heat generation/absorption is taken into account. The fluid viscosity is assumed to vary as an inverse linear function of temperature. A generalized similarity transformation is used to reduce the governing partial differential equations to a system of non-linear coupled ordinary differential equations, and is solved numerically by using a finite difference scheme. The numerical results concerned with the velocity, temperature and concentration distributions as well as the skin-friction coefficient and the Nusselt number for various values of the dimensionless parameters of interest are obtained. Some important findings reported in this paper reveal that the effect of thermal radiation and heat generation/absorption have significant role in controlling the rate of heat transfer in the boundary layer region.
\end{abstract}

Keywords: Thermal radiation, Chemical reaction, Variable viscosity, Inclined sheet.

\section{NOMENCLATURE}

$\begin{array}{ll}a, T_{r} & \text { constants } \\ B_{0} & \text { constant magnetic field strength } \\ C & \text { dimensional concentration variable } \\ C_{w} & \text { concentration at the sheet } \\ C_{f} & \text { skin-friction coefficient } \\ C_{p} & \text { specific heat at constant pressure } \\ D & \text { molecular diffusivity } \\ E_{C} & \text { Eckert number } \\ f & \text { dimensionless stream function } \\ G r_{t} & \text { Grashof number } \\ G r_{C} & \text { Modified Grashof number } \\ g_{0} & \text { acceleration due to gravity } \\ K & \text { mean absorption coefficient } \\ k & \text { thermal conductivity } \\ k_{0} & \text { reaction rate constant } \\ M & \text { magnetic parameter } \\ N r & \text { thermal radiation parameter } \\ N u & \text { Nusselt number } \\ n & \text { power index } \\ \mathrm{Pr} & \text { Prandtl number } \\ Q & \text { heat source/sink }\end{array}$

$\begin{array}{ll}\mathrm{Re}_{-} \mathrm{x} & \text { local Reynolds number } \\ S c & \text { Schmidt number } \\ T & \quad \text { dimensional temperature variable } \\ T_{\infty} & \text { free stream temperature } \\ T_{w} & \text { temperature at the sheet } \\ U_{w} & \text { surface velocity of the sheet } \\ u, v & \text { dimensional velocity components } \\ \alpha & \text { angle of inclination } \\ \beta & \text { nonlinear stretching parameter } \\ \beta_{t} & \text { thermal expansion coefficient } \\ \beta_{C} & \text { concentration expansion coefficient } \\ \lambda & \text { heat generation/ absorption parameter } \\ \sigma & \text { Stefan-Boltzmann constant } \\ \sigma & \text { electrical conductivity } \\ \psi & \text { dimensional stream function } \\ \eta & \text { non-dimensional distance } \\ \theta & \text { dimensionless temperature } \\ \varphi & \text { dimensionless concentration } \\ \mu & \text { coefficient of viscosity } \\ \rho_{\infty} & \text { density of the free stream } \\ \theta_{r} & \text { viscosity parameter }\end{array}$


$q_{r} \quad$ heat flux

\section{INTRODUCTION}

The boundary layer flow over a nonlinear stretching sheet has been the recent topic of researchers and engineers, because of its many engineering and industrial applications. The investigation of the boundary-layer flows and heat transfer of an incompressible fluid over an inclined stretching surface has also many important applications such as the extrusion of plastic sheets from a die, the boundary layer along a liquid film condensation process, gas turbines, MHD power generators, the cooling process of metallic plate in a cooling bath, in the glass and polymer industries, flight magnetohydrodynamics as well as in the field of planetary magnetosphere, aeronautics and chemical engineering. The rate of heat transfer at the stretching sheet plays a vital role to obtain better quality of the final product. If the temperature of the surrounding fluid becomes high, then the thermal radiation effect has a significant impact in the case of space technology. The effects of mass transfer with chemical reaction on the boundary layer flow has enormous applications in chemical engineering processes such as to enhanced oil recovery, packed-bed catalytic reactors, solidification of binary alloy as well as catalytic surface reactions in hydrodynamic flows.

Sakiadis (1961) initiated the study of boundary layer flow on a continuous moving surface. Later on Crane (1970) extended this problem to a stretching sheet whose surface velocity varies linearly with the distance $\mathrm{x}$ from the fixed point. Consequently Gupta and Gupta (1977) examined the heat and mass transfer over a linear stretching sheet subjected to suction/blowing. The influence of uniform magnetic field on the flow of an electrically conducting fluid past a stretching sheet have been investigated by Pavlov (1974), Andersson (1992), Watanabe and Pop (1993, 1995), Shit (2009), Prasad et al. (2010) and Shit and Haldar (2012a). Afify (2004) investigated the chemical reaction on free convective flow and mass transfer of a viscous, incompressible and electrically conducting fluid over a stretching sheet in the presence of a uniform transverse magnetic field. Misra and Shit (2008) extensively studied on electrically conducting fluid flow and heat transfer in a parallel plate channel with stretching walls in the presence of a magnetic field applied externally. Two-dimensional unsteady MHD flow of a viscous fluid between two moving parallel plates has been investigated by Sweet et al. (2011). Jafar et al. (2012) carried out the steady magnetohydrodynamic (MHD) laminar boundary layer flow of a viscous and incompressible electrically conducting fluid near the stagnation point on a horizontal stretching or shrinking surface, with variable surface temperature and a constant magnetic field applied normal to the surface of the sheet.

Recently, a new idea is added to the study of boundary layer fluid flow and heat transfer is the consideration of the effect of thermal radiation and temperature dependent viscosity. Many processes in engineering applications occur at high temperature and the radiated $\gamma \quad$ chemical reaction parameter

heat transfer becomes very important for the design of the pertinent equipment. Motivated by these assumptions, Shit and Haldar (2011a, 2012b) examined the combined effects of thermal radiation and hall current on momentum, heat and mass transfer in the laminar boundary layer fluid flow over an inclined permeable stretching sheet with variable viscosity. Tak et al. (2010) investigated the interaction of radiation with free convection in Darcian porous media by taking into account the Soret and Dufour's effects. Nonlinear hydromagnetic flow over a stretching surface with prescribed heat and mass flux embedded in a porous medium have analyzed analytically by Anjalidevi and Kayalvizhi (2013). Mukhopadhaya et al. (2005) investigated the problem of MHD boundary layer flow over a heated stretching sheet with variable viscosity. But, Salem (2007) investigated the effect of variable viscosity on MHD viscoelastic fluid flow and heat transfer over a stretching sheet without considering the effect of thermal radiation. Moreover, an analytical solution was obtained by Fang and Zhang (2010) for the flow and heat transfer over a linear shrinking sheet with mass transfer, wherein the effects of thermal radiation and variable thermal conductivity have been neglected. However, all the above studies are restricted in a linear stretching sheet.

Vajravelu and Cannon (2006) numerically examined the effects of nonlinearly stretching sheet on the flow characteristics of viscous incompressible fluid. Prasad et al. (2013) have investigated the effects of internal heat generation/absorption, thermal radiation, magnetic field and temperature-dependent thermal conductivity on the flow and heat transfer characteristics of a Non-Newtonian fluid over a stretching sheet. To characterize the non-Newtonian fluid behavior they have used upper convected Maxwell (UCM) fluid model. The numerical results for the physical variables have been obtained by using finite difference scheme along with the Keller-Box method. Mostafa et al. (2011) carried out the magnetohydrodynamic boundary layer flow and heat transfer of an electrically conducting micropolar fluid over a nonlinear stretching surface with variable wall heat flux in the presence of heat generation/absorption. Shit and Haldar (2011b) numerically investigated the effects of thermal radiation and variable fluid properties on the MHD fluid flow over a nonlinear porous shrinking sheet.

In this paper, we study the effects of thermal radiation and chemical reaction on the MHD fluid flow over a non-linear inclined stretching sheet with variable viscosity in the presence of heat generation /absorption. The viscous dissipation and buoyancy effects are taken into account in a situation when there is a temperature dependent viscosity. The present problem pertains to a situation in which the chemical reaction takes place. Since the governing differential equations are highly non-linear, Newton's linearization method is used followed by the finite difference scheme to obtain numerical solutions. The numerical results of the flow characteristics are presented graphically. 


\section{MATHEMATICAL FORMULATION AND FLOW ANALYSIS}

Let us consider the steady two-dimensional MHD flow and heat transfer along with the chemical reaction phenomenon of an incompressible, viscous and electrically conducting fluid past over an inclined nonlinear stretching sheet.

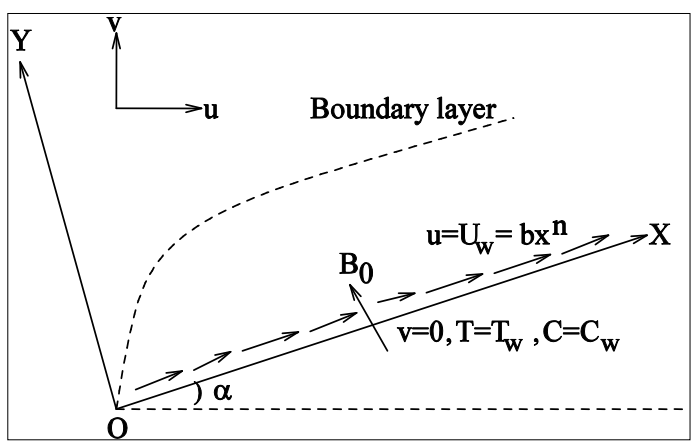

Fig. 1. A physical sketch of the problem

A uniform strong magnetic field of strength $B_{0}$ is imposed along the perpendicular to the sheet (Fig.1). The $\mathrm{x}$-axis is assumed to be the direction of the flow and $y$-axis is normal to it. The temperature and the species concentration are maintained at a prescribed constant value $T_{w}$ and $C_{w}$ at the sheet and $T_{\infty}$ and $C_{\infty}$ are the fixed values far away from the sheet.

The continuous stretching surface is assumed to have a Power-law velocity $u=U_{w}=b x^{n}$, where $b>0$ is a constant, $x$ denotes the distance from the slit, $n$ is the power index and $U_{w}$ represents the surface velocity of the sheet. We consider that electrically conducting fluid is influenced by the applied magnetic field $B(x)$ normal to the stretching sheet. The strength of the magnetic field $B(x)$ is taken as $B(x)=B_{0} x^{\frac{n-1}{2}}$ in which $B_{0}$ represents the constant magnetic field strength perpendicular to the sheet. We assume that the induced magnetic field produced by the motion of an electrically conducting fluid is negligible, since there is no electric field because of the negligible polarization of charges. The effect of induced magnetic field can also be neglected because of the low electrical conductivity of the fluid which in turn produces low magnetic Reynolds number.

According to the research conducted by Lai and Kulacki (1990), the fluid viscosity is assumed to vary as a reciprocal of a linear function of the temperature given by

$\frac{1}{\mu}=\frac{1}{\mu_{\infty}}\left[1+\gamma_{0}\left(T-T_{\infty}\right) \quad\right.$ i.e. $\quad \frac{1}{\mu}=a\left(T-T_{r}\right)$

where $a=\frac{\gamma_{0}}{\mu_{\infty}}, T_{r}=T_{\infty}-\frac{1}{\gamma_{0}}$

In Eq. (1), both $a$ and $T_{r}$ are constants and their values depend on the thermal property of the fluid, i.e., $\gamma_{0}$. In general $a>0$ represents for liquid, whereas $a<0$ represents gas.

By assuming Rosseland approximation for radiation, the radiative heat flux $q_{r}$ is given by $q_{r}=-\frac{4 \sigma^{*}}{3 K} \frac{\partial T^{4}}{\partial y}$

where $\sigma^{*}$ is defined as the Stefan-Boltzman constant and $K$ is the mean absorption coefficient. The temperature differences within the flow are so small under the consideration that $\boldsymbol{T}^{4}$ may be expressed as linear function of the temperature. Expanding $T^{4}$ in a Taylor series about $T_{\infty}$ and neglecting the higher order terms, we obtain

$T^{4} \cong 4 T_{\infty}^{3} T-3 T_{\infty}^{4}$

Substituting Eq. (4) in Eq. (3) yields

$\frac{\partial q_{r}}{\partial y}=-\frac{16 \sigma^{*} T_{\infty}^{3}}{3 K^{*}} \frac{\partial^{2} T}{\partial y^{2}}$

Owing to the above mentioned assumptions, the boundary layer flow over a non-linear stretching sheet is governed by the following system of equations

$\frac{\partial u}{\partial x}+\frac{\partial v}{\partial y}=0$

$\rho_{\infty}\left(u \frac{\partial u}{\partial x}+v \frac{\partial u}{\partial y}\right)=\frac{\partial}{\partial y}\left(\mu \frac{\partial u}{\partial y}\right)$

$+\rho_{\infty} g_{0}\left[\beta_{t}\left(T-T_{\infty}\right)+\beta_{c}\left(c-c_{\infty}\right)\right] \cos \alpha-\sigma B(x)^{2} u$

$\rho_{\infty} C_{p}\left(u \frac{\partial T}{\partial x}+v \frac{\partial T}{\partial y}\right)=k \frac{\partial^{2} T}{\partial y^{2}}+\sigma B(x)^{2} u^{2}$

$+\mu\left(\frac{\partial u}{\partial y}\right)^{2}-Q\left(T-T_{\infty}\right)-\frac{\partial q_{r}}{\partial y}$

$u \frac{\partial C}{\partial x}+v \frac{\partial C}{\partial y}=D \frac{\partial^{2} C}{\partial y^{2}}-K_{0}\left(C-C_{\infty}\right)$

where $u$ and $v$ are the velocity components along the $x$ and $y$ directions respectively, $\mu$ is the coefficient of viscosity, $g_{0}$ the acceleration due to gravity, $\beta_{t}$ the coefficient of thermal expansion, $\beta_{c}$ the coefficient of expansion with concentration, $\alpha$ the angle of inclination of the stretching sheet with the horizontal line. $T$ and $C$ are the temperature and concentration variable respectively, $D$ the thermal molecular diffusivity, $k_{0}$ is the reaction rate constant, $C_{p}$ is the specific heat at constant pressure, $k$ is the thermal conductivity, $T_{\infty}$ and $\rho_{\infty}$ are the free stream temperature and density. The term $Q\left(T-T_{\infty}\right)$ on the right hand side of Eq. (7) represents the amount of heat generated or absorbed per unit volume. The source term represents the heat source when $Q<0$ and heat sink when $Q>0$.

The boundary conditions for the present problem can be written as

$u=U_{w}=b x^{n}, \quad v=0, \quad T=T_{w}, \quad C=C_{w}$ at $y=0$

$u \rightarrow 0, \quad T \rightarrow T_{\infty}, \quad C \rightarrow C_{\infty}$ as $y \rightarrow \infty$

Let us introduce the following similarity transformations as 


$$
\begin{aligned}
& \psi(x, y)=\sqrt{\frac{2 v b x^{n+1}}{n+1}} f(\eta), \\
& u=\frac{\partial \psi}{\partial y}=b x^{n} f^{\prime}(\eta), \\
& v=-\frac{\partial \psi}{\partial x}=-\sqrt{\frac{b v(n+1)}{2}} x^{\frac{n-1}{2}}\left\{f(\eta)+\frac{n-1}{n+1} \eta f^{\prime}(\eta)\right\}, \\
& \eta=\frac{\sqrt{b(n+1)}}{2 v} x^{\frac{n-1}{2}} y, \\
& \theta(\eta)=\frac{T-T_{\infty}}{T_{w}-T_{\infty}} \\
& \varphi(\eta)=\frac{C-C_{\infty}}{C_{w}-C_{\infty}}
\end{aligned}
$$

where $\$$ and $f$ represent the dimensional and nondimensional stream function respectively, $v=\frac{\mu}{\rho_{\infty}}$ the kinematic viscosity, $n$ is a similarity space variable in non-dimensional form, $\theta$ and $\varphi$ are the dimensionless temperature and concentration.

Clearly, the continuity Eq. (5) is satisfied by $u$ and $v$ defined in Eq. (11). Substitution of Eq. (11) in Eq. (6) to Eq. (8) yields

$$
\begin{aligned}
& f^{\prime \prime \prime}+\beta \frac{\theta-\theta_{r}}{\theta_{r}}\left(f^{\prime}\right)^{2}-\frac{\theta-\theta_{r}}{\theta_{r}} f f^{\prime \prime}- \\
& \frac{\theta^{\prime}}{\theta-\theta_{r}} f^{\prime \prime}-\frac{\theta-\theta_{r}}{\theta_{r}}(2-\beta) \cos \cos \alpha\left[G r_{t} \theta+G r_{c} \varphi\right]+ \\
& M\left(\frac{\theta-\theta_{r}}{\theta_{r}}\right) f^{\prime}=0
\end{aligned}
$$

$\left(3 N_{r}+4\right) \theta^{\prime \prime}+3 N_{r} P_{r} f \theta^{\prime}+3 E_{c} N_{r} P_{r} M\left(f^{\prime}\right)^{2}-$

$3 \lambda N_{r} P_{r} \theta-\left(\frac{\theta_{r}}{\theta-\theta_{r}}\right) 3 N_{r} P_{r} E_{c}\left(f^{\prime \prime}\right)^{2}=0$

$\phi^{\prime \prime}+S c\left(f \phi^{\prime}-\gamma \phi\right)=0$

and the transformed boundary conditions are given by

$$
\begin{aligned}
& f^{\prime}(\eta)=1, f(\eta)=0, \theta(\eta)=1, \phi(\eta)=1, \text { at } \eta=0 \\
& f^{\prime}(\eta) \rightarrow 0, \theta(\eta) \rightarrow 0, \phi(\eta) \rightarrow 0 \quad \text { when } \eta \rightarrow \infty
\end{aligned}
$$

where primes denote differentiation with respect to $\eta$ only.

The dimensionless parameter appeared in Eq. (12) to Eq. (14) are defined as $\theta_{r}=\frac{T_{r}-T_{\infty}}{T_{w}-T_{\infty}}=-\left(\frac{1}{\gamma_{0}\left(T_{w}-T_{\infty}\right)}\right)$ the viscosity parameter, $\quad M=\frac{2 \sigma B_{0}^{2}}{\rho_{\infty} b(n+1)}$ the magnetic parameter, $\operatorname{Pr}=\frac{\rho C_{p^{v}}}{k}$ the Prandtl number,

$\gamma=\frac{2 k_{0}}{b(n+1) x^{n-1}}\left(C_{w}-C_{\infty}\right)$ the non-dimensional chemical reaction parameter, $G r_{t}=\frac{g_{0} \beta_{t}\left(T_{w}-T_{\infty}\right)}{b^{2} x^{2 n-1}}$ the local Grashof number, $G r_{C}=\frac{g_{0} \beta_{c}\left(C_{w}-C_{\infty}\right)}{b^{2} x^{2 n-1}}$ the local modified
Grashof number, $\quad N r=\frac{k K}{4 T_{\infty}^{3} \sigma^{*}}$ the thermal radiation parameter, $\quad E c=\frac{U_{w}{ }^{2}}{C_{p}\left(T_{w}-T_{\infty}\right)}$ the Eckert number, $\lambda=\frac{2 Q}{b(n+1) \rho_{\infty} C_{p^{x}} x^{n-1}}$ the heat generation or absorption parameter, $\quad S c=\frac{\mu}{\rho_{\infty} D}$ the Schmidt number and $\beta=\frac{2 n}{n+1}$ the non-linear stretching parameter.

It is worthwhile to mention here that $\theta_{r}$ is negative for liquids and positive for gases. The elimination of $\gamma_{0}$ between the Eq. (1) and the definition of $\theta_{r}$ gives rise $\mu=\frac{\mu_{\infty}}{\left(1-\frac{\theta}{\theta_{r}}\right)}$ to.

From this relation it is obvious that when $-\theta_{r} \rightarrow \infty$ then $\mu \rightarrow \mu_{\infty}$ i.e. the viscosity variation in the boundary layer is negligible. However, the viscosity variation is more significant as $-\theta_{r} \rightarrow 0$ when $M=0, \beta=1$ and $G r_{t}=G r_{c}=0$, the present problem reduces to hydrodynamic boundary layer flow past a stretching sheet whose analytical solutions put forwarded by Crane [2] as follows

$$
f(\eta)=1-e^{-\eta} \text { i.e. } f^{\prime}(\eta)=e^{-\eta}
$$

With an aim to test the accuracy of our numerical result for axial velocity $f^{\prime}(\eta)$, we have compared our result with this analytical solution.

The important characteristics of the present study are the local-skin-friction coefficient $C_{f}$ and the local Nusselt number $N_{u}$ defined by

$$
\sqrt{\mathrm{Re}_{-} \mathrm{x}} C_{f}=-\left(\frac{\theta_{r}}{1-\theta_{r}}\right) \sqrt{\frac{1}{2-\beta} f^{\prime \prime}(0)}
$$

where $C_{f}=\frac{\tau_{w}(x)}{\rho U_{w}^{2}}$ with

$\tau_{w}=\mu\left(\frac{\partial u}{\partial y}\right)_{y=0}$

$\frac{N u}{\sqrt{\operatorname{Re}_{-} \mathrm{x}}}=-\sqrt{\frac{1}{2-\beta}} \theta^{\prime}(0)$,

where $N u=\frac{x q_{w}}{k_{\infty}\left(T_{w}-T_{\infty}\right)}$, with

$q_{w}=-k_{w}\left(\frac{\partial T}{\partial y}\right)_{y=0}$, 
where $\mathrm{Re}_{-} \mathrm{x}=\frac{U x}{v}$ is defined as the local Reynolds number.

\section{RESULTS AND DISCUSSION}

The system of coupled and non-linear ordinary differential Eq. (12) to Eq. (14) along with the boundary conditions Eq. (15) and Eq. (16) have been solved numerically by employing a finite difference scheme. We used Newton's linearization method (Cebeci and Cousteix (1999), Misra and Shit (2009a, 2009b) to linearize the discretized equations. The essential features of this technique are that it is based on a finite difference scheme, which has better stability, simple, accurate and more efficient. Finite difference technique leads to a system which is tridiagonal and therefore speedy convergence as well as economical memory space to store the coefficients. The computational work has been carried out by taking $\delta_{\eta}=0.0125$ and further reduction in $\delta_{\eta}$ does not bring about any significant change. For the purpose of numerical computation it is necessary to assign some valid numerical values to the physical parameters involved in the problem. In this connection, the following data have been used (Naduvinamani and Santosh 2011; Watanabe and Pop 1993, 1995; Afify 2004 and Salem 2007):

$M \in[0,6] ; \theta_{r}=-1,-2,-5,-10 ; \beta=-1,-0.5,0.5,1 ;$

$\alpha=0, \frac{\pi}{6}, \frac{\pi}{4}, \frac{\pi}{3} ; \operatorname{Pr}=0.71,1,5,7 ; N r=0.5,1,5,7 ;$

$\lambda=-0.5,-0.3,0,0.5,1,2 ; \gamma=0.5,1,1.5 ; S c=0.5,1,1.5$

The use of non-Newtonian fluid as lubricants is of growing interest for the motion of fluids that contain coarse structures and fibers such as colloidal fluids and liquid crystals. In view of this an attempt has been made to study the micropolar fluids by treating it as lubricants. The quality of lubricating oils and lubricants is attained through purification and manufacturing processes in addition of certain chemical agents. The major consideration in selecting oil for a particular application is viscosity and its variation depends on temperature. However, we used wide range of the values of physical parameters stated above for an illustrative example in order to apply to a greater extend.

Figure 2 shows that our numerical results are in complete agreement with the analytical results of Crane (1970). Figures 3-7 give the variation of axial component of velocity $f^{\prime}(\eta)$ along with the perpendicular distance $\eta$ from the sheet for different values of the physical parameters of interest. We observe from Fig. 3 that the axial velocity in the boundary layer region decreases gradually with the increase of the magnetic field strength. This may attribute to the Lorentz force that arises due to the application of an external magnetic field in an electrically conducting fluid. This force has a tendency to slow down the motion of the fluid and thereby decreases momentum boundary layer thickness. It is interesting to note from Fig. 4 that the axial velocity also decreases with the increase of the stretching parameter $\beta$.

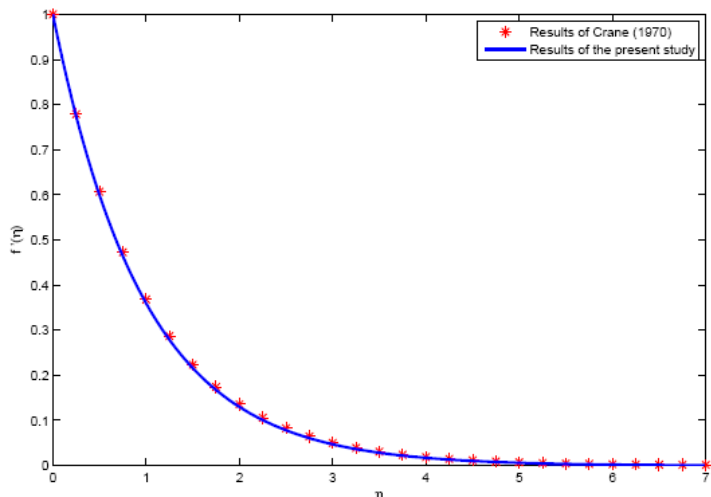

Fig. 2. Comparison of axial velocity with the analytical results of Crane (1970) without applying any external body force

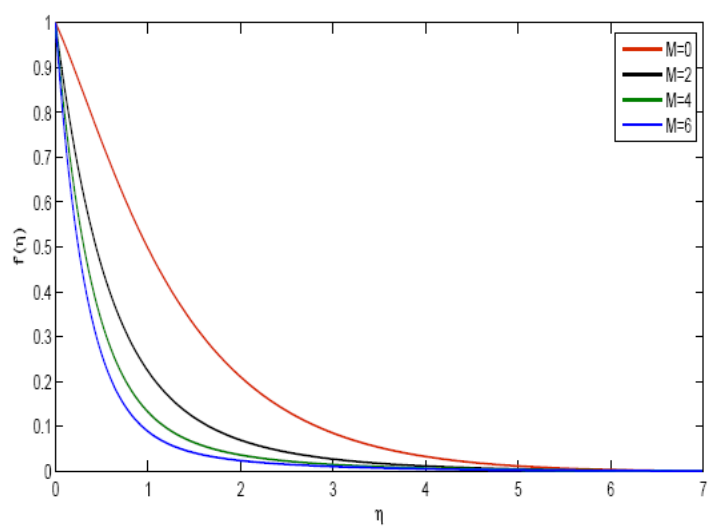

Fig. 3. Variation of $f^{\prime}(\eta)$ with $\eta$ for different values of $\mathrm{M}$ (with $\theta_{\mathrm{r}}=-2, \beta=-1, \alpha=\pi / 4, \operatorname{Pr}=1, \mathrm{Nr}=1, \lambda=1$, $\gamma=0.5)$

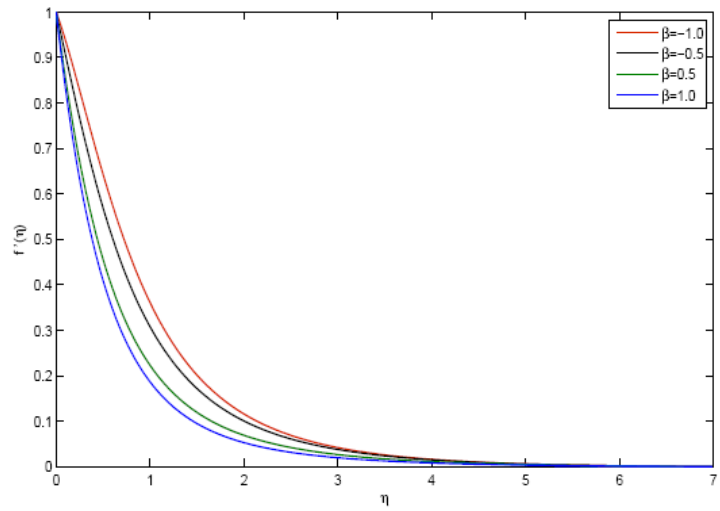

Fig. 4. Variation of $f^{\prime}(\eta)$ with $\eta$ for different values of $\beta$ (with $\mathrm{M}=2, \theta_{\mathrm{r}}=-2, \alpha=\pi / 4, \mathrm{Pr}=1, \mathrm{Nr}=1, \lambda=1, \gamma=$ $0.5)$

Therefore, non-linearity of the stretching sheet has a reducing effect on the momentum boundary layer thickness. In the present study $\beta=1$ corresponds to the problem of linear stretching sheet as studied earlier by Shit and Haldar (2011b). Figure 5 depicts the variation of axial velocity for different angle of inclination. It shows that the axial velocity decreases with the increase of the inclination angle $\alpha$ of stretching sheet. It is interesting to note from Fig. 6 that the axial velocity in the boundary layer region decreases with the increasing values of the heat generation/ absorption parameter $\lambda$. 
However, the viscosity parameter $\theta_{\mathrm{r}}$ has increasing effect on the momentum boundary layer thickness as shown in Fig. 7.

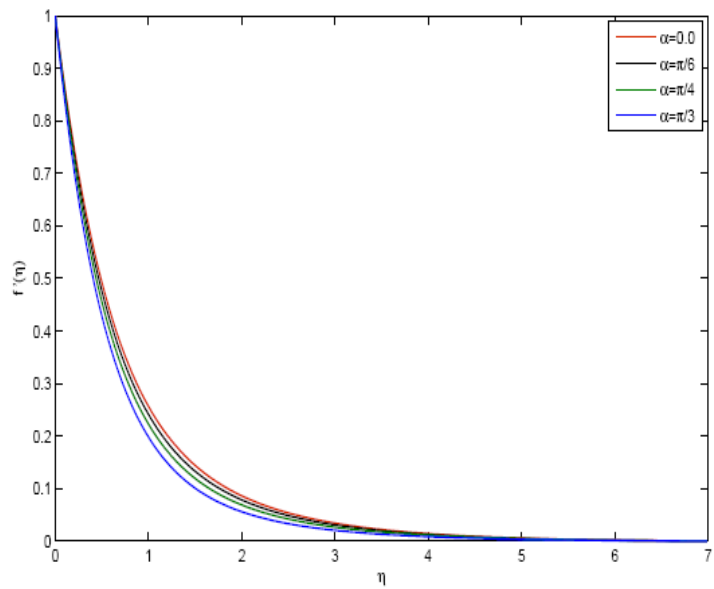

Fig. 5. Variation of $f^{\prime}(\eta)$ with $\eta$ for different values of $\alpha\left(\right.$ with $\mathrm{M}=2, \theta_{\mathrm{r}}=-2, \beta=-1, \mathrm{Pr}=1, \mathrm{Nr}=1, \lambda=1, \gamma$ $=0.5$ )

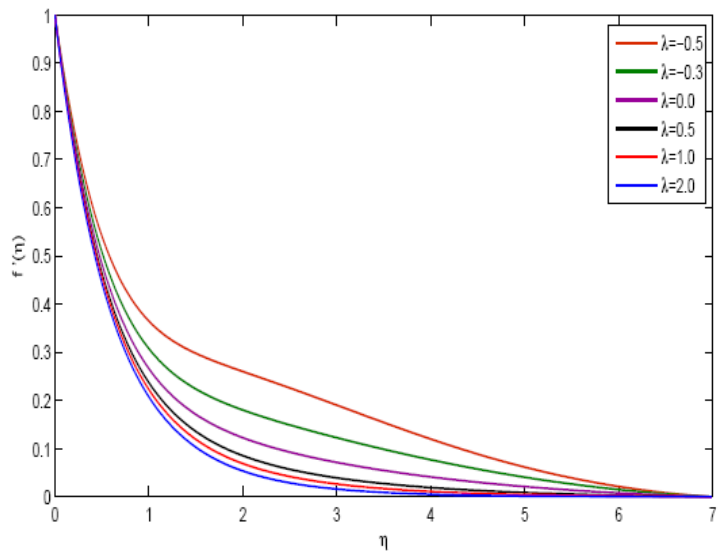

Fig. 6. Variation of $f^{\prime}(\eta)$ with $\eta$ for different values of $\lambda\left(\right.$ with $\mathrm{M}=2, \theta_{\mathrm{r}}=-2, \beta=-1, \alpha=\pi / 4, \mathrm{Pr}=1, \mathrm{Nr}=1, \gamma$ $=0.5$ )

Figures 8-10 illustrate the distribution of dimensionless temperature $\theta(\eta)$ along the height from the stretching sheet for different values of $\lambda, \mathrm{Nr}$ and Pr. We observe from Fig. 8 that the temperature at the sheet gradually decreases with the increase of the heat absorption parameter $\lambda>0$, whereas, the temperature increases initially up to a certain height above the sheet and beyond which it decreases monotonically for heat generation parameter $(\lambda<0)$. It is also observed that the heat generation/absorption parameter has dual characteristics in the region of thermal boundary layer. Fig. 9 shows that the temperature decreases with the increase of the thermal radiation parameter $\mathrm{Nr}$. It is interesting to note from this figure that the temperature decreases significantly up to a certain value i.e., $\mathrm{Nr}=5$, beyond which no change is observed. The effect of Prandtl Pr on the heat transfer is shown in Fig. 10. It reveals that the temperature is also significantly decreases with the increase of the Prandtl number Pr. The distribution of concentration species for different values of the chemical reaction parameter and the Schmidt number Sc are shown in Fig. 11 and Fig.
12 respectively. The concentration of species in the boundary layer decreases for increasing values of both the parameters $\gamma$ and Sc. Thus these two parameters are responsible for the reducing of concentration boundary layer thickness.

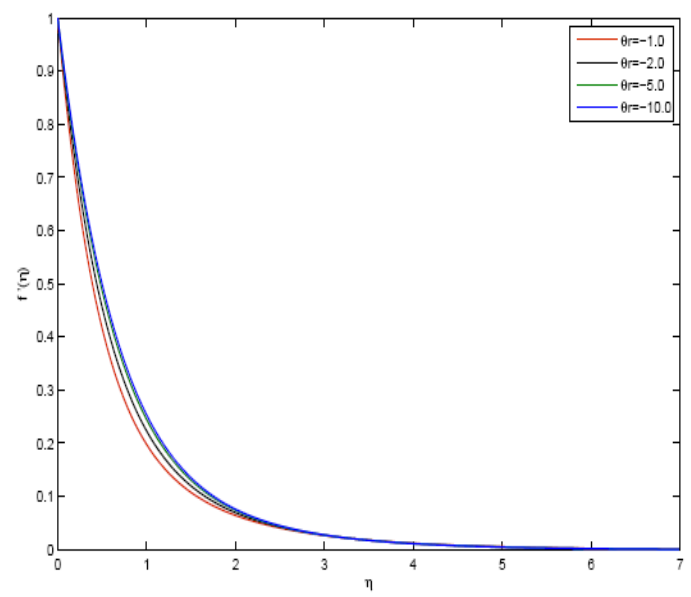

Fig. 7. Variation of $f^{\prime}(\eta)$ for different values of viscosity parameter $\theta_{\mathrm{r}}($ with $\mathrm{M}=2, \beta=-1, \alpha=\pi / 4, \operatorname{Pr}=$ $1, \mathrm{Nr}=1, \lambda=1, \gamma=0.5$ )

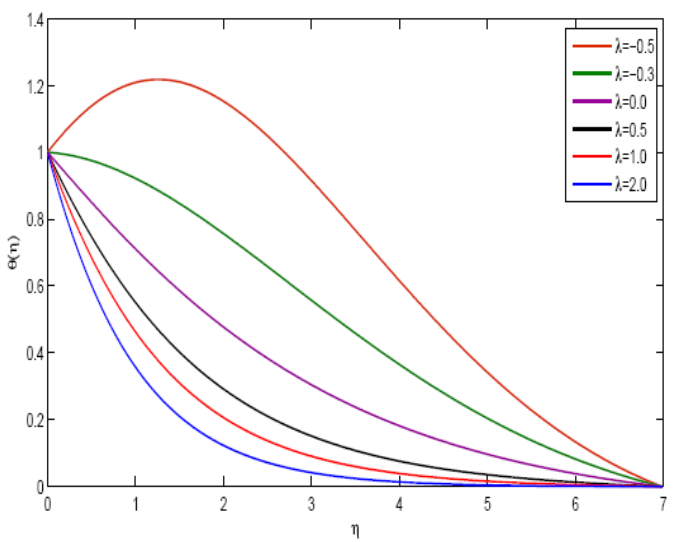

Fig. 8. Distribution of dimensionless temperature $\theta(\eta)$ for different values of $\lambda$ (with $M=2, \theta_{\mathrm{r}}=-2, \beta=-1, \alpha$ $=\pi / 4, \mathrm{Pr}=1, \mathrm{Nr}=1, \gamma=0.5$ )

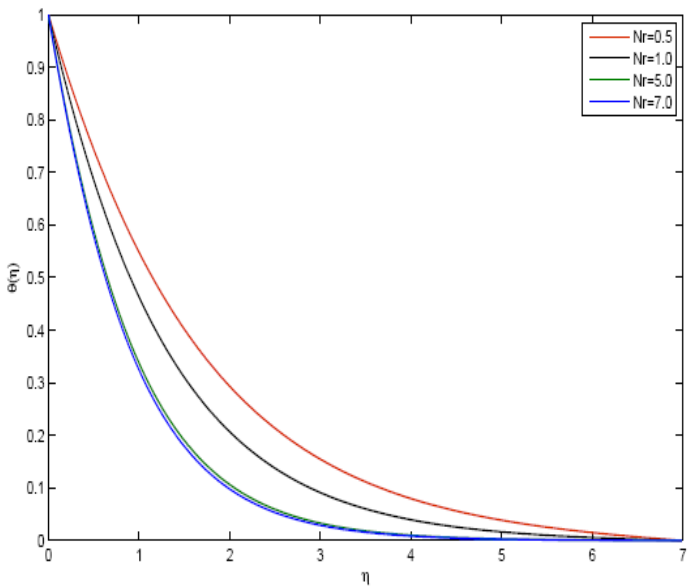

Fig. 9. Distribution of dimensionless temperature $\theta(\eta)$ for different values of thermal radiation parameter $\operatorname{Nr}\left(\right.$ with $\mathrm{M}=2, \theta_{\mathrm{r}}=-2, \beta=-1, \alpha=\pi / 4, \operatorname{Pr}=1, \lambda=1, \gamma=$ $0.5)$ 


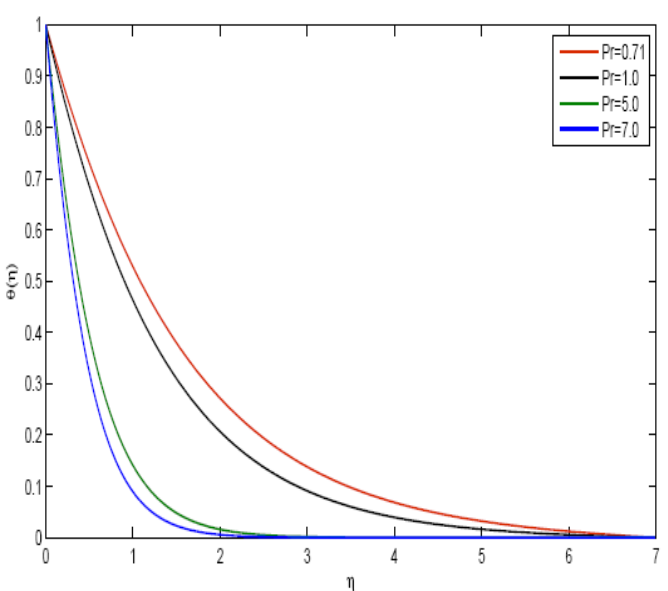

Fig. 10. Distribution of dimensionless temperature $\theta(\eta)$ for different values of $\operatorname{Pr}\left(\right.$ with $M=2, \theta_{\mathrm{r}}=-2, \beta=$ $1, \alpha=\pi / 4, \mathrm{Nr}=1, \lambda=1, \gamma=0.5$ )

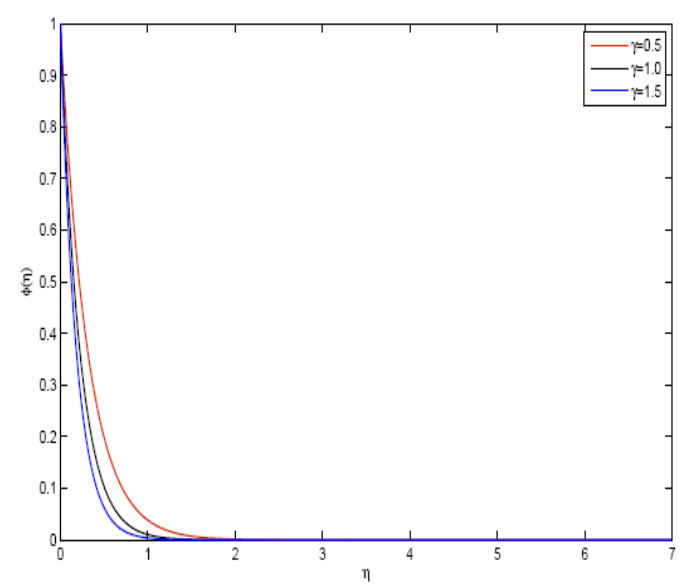

Fig. 11. Concentration profiles for different values of chemical reaction parameter $\gamma\left(\right.$ with $\mathrm{M}=2, \theta_{\mathrm{r}}=-2, \beta=$ $1, \alpha=\pi / 4, \operatorname{Pr}=1, \mathrm{Nr}=1, \lambda=1$ )

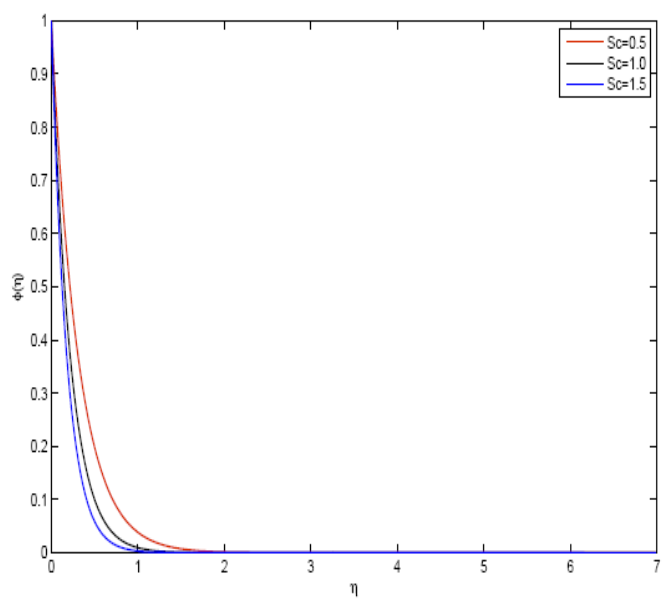

Fig. 12. Concentration profiles for different values of Schmidt number $S c$ (with $M=2, \theta_{\mathrm{r}}=-2, \beta=-1, \alpha=\pi / 4$, $\operatorname{Pr}=1, \mathrm{Nr}=1, \lambda=1, \gamma=0.5)$

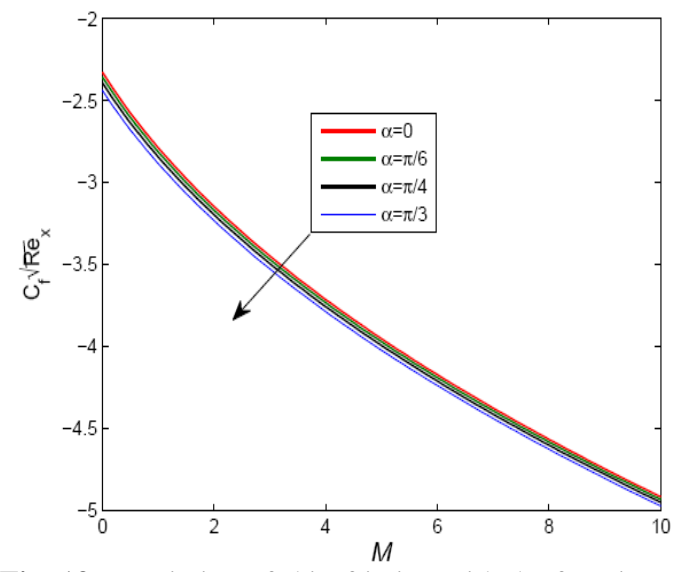

Fig. 13. Variation of skin-friction with the function of $M$ for different values of $\alpha$ (with $\theta_{\mathrm{r}}=-2, \beta=-1, \operatorname{Pr}=1$, $\mathrm{Nr}=1, \lambda=1, \gamma=0.5)$

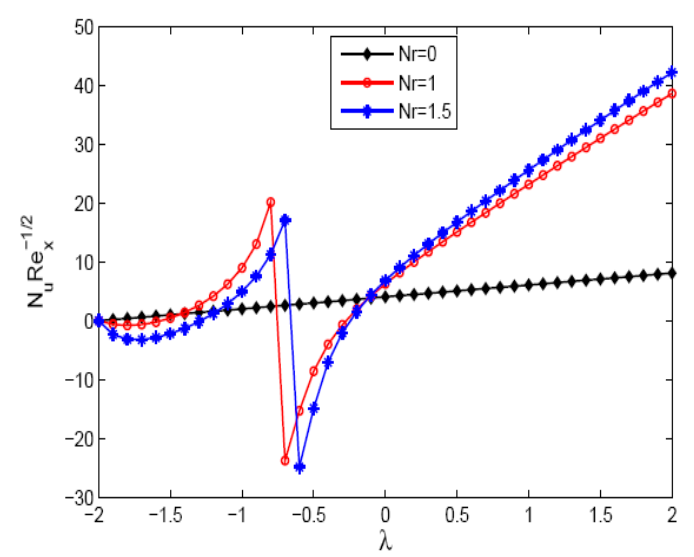

Fig. 14. Variation of Nusselt number with the function of $\lambda$ for different values of $\mathrm{Nr}$ (with $\mathrm{M}=2, \theta_{\mathrm{r}}=-2, \beta=$ -

$$
1, \alpha=\pi / 4, \operatorname{Pr}=1
$$

Figure 13 illustrates the variation of skin-friction $C_{f} \sqrt{\mathrm{Re}_{x}}$ as the function of Magnetic parameter M for different angle of inclination of the sheet. It reveals that the skin-friction decreases monotonically with increasing magnetic field strength. The skin-friction also decreases with the increase of the inclination angle of the sheet, but the change observed is minimal. For the sake of brevity, it has been observed that the skinfriction coefficient has no change in the absence of thermal radiation. Figure 14 represents the variation of Nusselt number $\frac{N u_{x}}{\sqrt{\operatorname{Re}_{x}}}$ that is the rate of heat transfer at the sheet as a function of heat generation/ absorption parameter $\lambda$ for different thermal radiation parameter $\mathrm{Nr}$. It is found that the rate of heat transfer gradually increases in the flow opposing region $(\lambda>0)$. However, an anomalous behavior of the Nusselt number is observed in the case of flow assisting region $(\lambda<0)$ in the presence of thermal radiation. It may be pointed out that there exists a critical value $\left(\lambda_{c}\right)$ of $\lambda<0$, where a rapid change of the rate of heat transfer takes place. Beyond this critical value of $\lambda$, the rate of heat transfer decreases as the heat generation parameter (negative values of $\lambda$ ) increases. It is interesting to note from this figure that the critical value $\lambda_{c}$ is different for different 
values of the thermal radiation parameter $\mathrm{Nr}$. But, no critical value exists in the absence of thermal radiation.

\section{CONCLUDING REMARKS}

The effect of thermal radiation and magnetohydrodynamic interaction of flow and heat transfer over an inclined non-linear stretching sheet have been the main concern of this investigation. The temperature dependent variable viscosity has also been the consideration of the present problem. The effect of various key parameters including the angle of inclination $(\alpha)$, magnetic parameter $(\mathrm{M})$, thermal radiation parameter $(\mathrm{Nr})$, heat generation/absorption parameter $\lambda$, non-linearity of the stretching sheet (i.e., the parameter $\beta$ ) as well as the chemical reaction parameter $\gamma$ are examined. The main findings of the present study may be concluded as follows:

- The axial velocity decreases in the boundary layer region for the increasing values of the angle of inclination, magnetic parameter, nonlinearity of the stretching sheet as well as the heat generation or absorption parameter $\lambda$.

- The increasing values of the thermal radiation parameter leads to the decreasing of thermal boundary layer thickness. Thus it may be used to increase the rate of cooling of the sheet.

- The interaction of concentration boundary layer decreases with the increase of the chemical reaction parameter $\gamma$.

- The skin-friction coefficient decreases with the increase of the magnetic parameter $M$ as well as the angle of inclination of the sheet.

There exists a critical value of $\lambda$ at which the dual characteristics of the rate of heat transfer is observed in the presence of thermal radiation. Therefore, the effect of thermal radiation plays an important role in controlling heat transfer at the sheet.

\section{ACKNOWLEDGEMENTS}

One of the authors (G. C. Shit) is thankful to UGC, New Delhi for supporting this investigation through UGC-DRS program of the Department of Mathematics, Jadavpur University, Kolkata.

\section{REFERENCES}

Afify, A.A (2004). MHD free-convective flow and mass transfer over a stretching sheet with chemical reaction. Heat and Mass Transfer, 40, 495-500.

Andersson, H.I., K. H. Bech and B.S. Dandapat (1992). Magnetohydrodynamic flow of a Power-law fluid over a stretching surface. International Journal of Non-Linear Mechanics, 27, 929-936.

Anjalidevi, S.P. and M. Kayalvizhi (2013). Nonlinear hydromagnetic flow with radiation and heat source over a stretching surface with prescribed heat and mass flux embedded in a porous medium. Journal of Applied Fluid Mechanics, 6(2), 157-165.

Cebeci, T. and J. Cousteix (1999). Modeling and computation of boundary-layer flows. SpringerVerlag.

Crane, L.J. (1970). Flow past a stretching sheet. Journal of Applied Mathematics and Physics(ZAMP),21, 645-647.

Fang, T. and J. Zhang (2010). Thermal boundary layers over a shrinking sheet: An analytical solution. ActaMechanica, 209, 325-343.

Gupta, P.S. and A.S. Gupta (1977). Heat and Mass Transfer on a stretching sheet with suction or blowing. The Canadian Journal of Chemical Engineering, 55, 744-746.

Jafar, K., R. Nazar, A. Ishak and I. Pop (2012). MHD Flow and Heat Transfer Over Stretching/Shrinking Sheets With External Magnetic Field, Viscous Dissipation and Joule Effects. The Canadian Journal of Chemical Engineering, 90, 1336-1346.

Lai, F.C. and F.A. Kulacki (1990). The effect of variable viscosity on convective heat transfer along a vertical surface in a saturated porous medium. International Journal of Heat and Mass Transfer, 33, 1028-1031.

Misra, J.C. and G.C. Shit (2009a). Biomagnetic viscoelastic fluid flow over a stretching sheet. Applied Mathematics and Computation, 210, 350361.

Misra, J.C., G.C. Shit and H.J. Rath (2008). Flow and heat transfer of a MHD viscoelastic fluid in a channel with stretching walls: Some applications to haemodynamics. Computers \& Fluids, 37, 1-11.

Misra, J. C. and G. C. Shit (2009b). Flow of a biomagnetic viscoelastic fluid in a channel with stretching walls. ASME Journal of Applied Mechanics, 76, 061006.

Mostafa, A., A. Mahmoud and S.E. Waheed (2011). MHD flow and heat transfer of a micropolar fluid over a nonlinear stretching surface with variable surface heat flux and heat generation. The Canadian Journal of Chemical Engineering, 89, 1408-1415.

Mukhopadhaya, S., G.C. Layek and S.A. Samad (2005). Study of MHD boundary layer flow over a heated stretching sheet with variable viscosity. International Journal of Heat and Mass Transfer, 48, 4460-4466.

Naduvinamani, N. B. and S. Santosh. (2011). Micropolar fluid squeeze film lubrication of finite porous journal bearing. Tribology International. 44, 409-416.

Pavlov, K. B. (1974). Magnetohydrodynamic flow of an incompressible viscous fluid caused by 
deformation of plane surface. Magnitnaya Gidrodinamika, 4, 146-147.

Prasad, K.V., P.S. Dattiand K. Vajravelu (2010). Hydromagnetic flow and heat transfer of a nonNewtonian power law fluid over a vertical stretching sheet. International Journal of Heat and Mass Transfer, 53, 879-888.

Prasad, K.V., K. Vajraveluand A. Sujatha (2013). Influence of internal heat generation/absorption, thermal radiation, magnetic field, variable fluid property and viscous dissipation on heat transfer characteristics of a Maxwell fluid over a stretching sheet. Journal of Applied Fluid Mechanics, 6(2), 249-256.

Sakiadis, B.C. (1961). Boundary layer behavior on continuous solid surfaces. American Institute of Chemical Engineering Journal (AIChE), 7, 26-28.

Salem, A.M. (2007). Variable viscosity and thermal conductivity effects on MHD flow and heat transfer in viscoelastic fluid over a stretching sheet. Physics letters A, 369, 315-322.

Shit, G.C. (2009). Hall effects on MHD free-convective flow and mass transfer over a stretching sheet. International Journal of Applied Mathematics and Mechanics, 5(8), 22-38.

Shit, G.C. and R. Haldar (2011a). Thermal radiation and Hall effect on MHD flow, heat and mass transfer over an inclined permeable stretching sheet. Thermal Science, 15, 195- 204.

Shit, G.C. and R. Haldar (2012b). Combined effects of thermal radiation and hall current on MHD freeconvective flow and mass transfer over a stretching sheet with variable viscosity. Journal of Applied Fluid Mechanics, 5(2), 113-121.
Shit, G.C. and R. Haldar (2011b). Effects of thermal radiation on MHD viscous fluid flow and heat transfer over nonlinear shrinking porous sheet. Applied Mathematics and Mechanics, 32, 677-688.

Shit, G. C. and R. Haldar (2012a). Thermal radiation effects on MHD viscoelastic fluid flow over a stretching sheet with variable viscosity. International Journal of Applied Mathematics and Mechanics, 8, 14-36.

Sweet, E., K. Vajravelu., R.A. Van Gordar and I. Pop (2011). Analytical solution for the unsteady MHD flow of a viscous fluid between moving parallel plates. Communications in Nonlinear Science and Numerical Simulation, 16, 266-273.

Tak, S.S., R. Mathur., R. K. Gehlotand A. Khan (2010). MHD free convection-radiation interaction along a vertical surface embedded in Darcian porous medium in presence of Soret and Dufour's effects. Thermal Science, 14, 137-145.

Vajravelu, K. and C.R. Cannon (2006). Fluid flow over a nonlinearly stretching sheet. Applied Mathematics and Computation, 181, 609-618.

Watanabe, T. and I. Pop (1993). Magnetohydrodynamics free-convection flow over a wedge in the presence of a transverse magnetic field. International Communications in Heat and Mass transfer, 20, 871-881.

Watanabe, T. and I. Pop (1995). Hall effects on magnetohydrodynamic boundary layer flow over a continuous moving flat plate. Acta Mechanica, $108,35-47$. 